\title{
Very late ischemic complications in flow-diverter stents: a retrospective analysis of a single-center series
}

\author{
Alexis Guédon, MD, ${ }^{1}$ Frédéric Clarençon, MD, PhD, ${ }^{1,2}$ Federico Di Maria, MD, ${ }^{1}$ \\ Charlotte Rosso, MD, PhD, ${ }^{2,3}$ Alessandra Biondi, MD, PhD, ${ }^{4}$ Joseph Gabrieli, MD, ${ }^{1,2}$ \\ Patricia Rojas, MD, ${ }^{5}$ Jacques Chiras, MD, ${ }^{1,2}$ and Nader Sourour, MD1
}

\begin{abstract}
Departments of ${ }^{1}$ Interventional Neuroradiology, ${ }^{3}$ Vascular Neurology, and ${ }^{5}$ Neuro-anesthesiology, Pitié-Salpêtrière Hospital; ${ }^{2}$ Paris VI University, Pierre et Marie Curie, Paris; and ${ }^{4}$ Department of Neuroradiology and Endovascular Therapy, Jean Minjoz University Hospital, Franche Comté University School of Medecine, Besançon, France
\end{abstract}

\begin{abstract}
OBJECTIVE The authors evaluate the rate and discuss the pathomechanisms of very late ( $\geq 4$-month) ischemic complications after flow-diverter stent (FDS) placement for intracranial aneurysms.

METHODS The authors retrospectively reviewed the clinical data of the patients treated at Pitié-Salpêtrière Hospital between January 2010 and September 2014, who underwent FDS placement for intracranial aneurysm. The patients received dual-antiplatelet therapy (clopidogrel and aspirin) 5 days before and 3-6 months after the procedure and then aspirin alone for 6-9 months. An ischemic complication was defined as a sudden focal neurological deficit documented on diffusion-weighted images.
\end{abstract}

RESULTS Eighty-six consecutive patients were included. Three (3.5\%) patients treated with the Pipeline embolization device experienced a delayed acute ischemic stroke (2 cases of perforator/side-wall branch infarction and 1 case of thromboembolic stroke) with an average delay of 384 days (4 months, 20 months, and 13 months, respectively). The aneurysm locations were the left superior cerebellar artery, the right anterior choroid artery, and the left internal carotid artery (paraclinoid segment), respectively. The complications occurred after the patients had completed the antiaggregation protocol, except for Patient 1, who was receiving aspirin alone because of a spontaneous hematoma. At the acute phase, no in-stent thromboses were found on digital subtraction angiography. In Patient 2, the treated anterior choroid artery was occluded 20 months after the procedure. In Patient 3, a focal stenosis (approximately 40\%) of the distal aspect of the FDS, probably caused by intimal hyperplasia, was seen.

CONCLUSIONS Very late ischemic complications after FDS treatment were observed in $3.5 \%$ of the cases in the authors' series, some of which occurred as late as more than 1 year after placement.

http://thejns.org/doi/abs/10.3171/2015.10.JNS15703

KEY WORDS flow-diverter stent; stroke; delayed complication; Pipeline embolization device; vascular disorders

$\mathrm{D}$ URING the past decade, the advent of flow-diverter stents (FDSs) has offered an alternative for the treatment of aneurysms of complex shapes (giant/ large, large-neck, and/or dissecting/fusiform aneurysms). FDSs are able to form a tight mesh, and the aim is to divert blood flow from the aneurysm sac toward the downstream artery and obtain the aneurysm sac thrombosis. Furthermore, FDSs induce the development of a neointima on the surface of the aneurysm's neck. FDSs have fundamentally changed the management approach from one of intracranial treatment using endosaccular materials to one of us- ing extraaneurysmal endoluminal devices. Because FDSs are relatively new devices, some of their associated complications are unpredictable, and the treatment of cerebral aneurysm with an FDS is not free of risk. In a recent metaanalysis in which data from 29 studies were pooled, Brinjikji et al. ${ }^{5}$ reported a procedure-related mortality rate of $4 \%(95 \%$ CI $3 \%-6 \%)$ and a morbidity rate of $5 \%(95 \%$ CI $4 \%-7 \%)$. Among postoperative complications, ischemic stroke and perforator infarction had reported incidences of $6 \%(95 \%$ CI $4 \%-9 \%)$ and $3 \%(95 \%$ CI $1 \%-5 \%)$, respectively. Very late ( $\geq 4$-month) ischemic complications

ABBREVIATIONS AChA = anterior choroid artery; DIPH = delayed ipsilateral parenchymal hemorrhage; DSA = digital subtraction angiography; DWI = diffusion-weighted imaging; FDS = flow-diverter stent; IQR = interquartile range; $m R S=$ modified Rankin Scale; NIHSS = NIH Stroke Scale; PED = Pipeline embolization device; SCA = superior cerebellar artery.

SUBMITTED April 1, 2015. ACCEPTED October 5, 2015.

INCLUDE WHEN CITING Published online January 29, 2016; DOI: 10.3171/2015.10.JNS15703. 
are rarely reported in the literature. ${ }^{11}$ The purpose of the present study was to report our experience with very late ischemic complications after FDS placement for the treatment of intracranial aneurysms.

\section{Methods}

We retrospectively reviewed the clinical data of patients consecutively treated in our institution between January 2010 and September 2014 for intracranial aneurysm by means of FDS placement.

All except for 3 patients received dual-antiplatelet therapy (clopidogrel and aspirin) 5 days before stent placement; these 3 patients were treated with ticagrelor because of clopidogrel resistance (determined by using a Multiplate [Roche Diagnostics] platelet-aggregation test). Dual antiaggregation was used for 3-6 months, and then aspirin alone was using for 6-9 months after the procedure. Patients resistant to clopidogrel were treated for 1 year with ticagrelor.

Each patient's chart was reviewed. Any sudden onset of a focal neurological deficit was systematically recorded. A very late ischemic complication was defined as a sudden focal neurological deficit associated with evidence of acute ischemic stroke on diffusion-weighted imaging (DWI). A 4-month cutoff was chosen because it corresponds to the intimal hyperplasia peak ${ }^{14}$ and to the relay area between double-antiplatelet therapy and aspirin therapy alone (1-6 months in most studies).

Patient demographics (i.e., age, sex, and comorbidities [blood hypertension, diabetes mellitus, hypercholesterolemia, and tobacco addiction]) and aneurysm characteristics (i.e., location, size [maximum diameter, neck size, and dome-to-neck ratio], and recanalization/first treatment) were systematically assessed. The pathomechanisms of the delayed acute ischemic stroke were systematically evaluated. After the diagnosis of acute ischemic stroke was made with MRI, each patient underwent digital subtraction angiography (DSA) to clarify the etiology of the stroke.

\section{Results}

Eighty-six consecutive patients who received an FDS were included in the study. The overall mean \pm SD and median (interquartile range [IQR]) angiographic (DSA and/or MR angiography) follow-ups were $15.7 \pm 11.8$ months and 13 months (8-21 months), respectively. The overall mean $\pm \mathrm{SD}$ and median (IQR) clinical follow-ups were $16.9 \pm$ 12.9 months and 14 months (10-22 months), respectively. The overall rate of ischemic complication was $5.8 \%$ (5 of 86). Among patients with complications, 3 (3.5\% [2 males and 1 female; average age 45 years]) presented with a very late DWI-documented ischemic complication; the average $( \pm$ SD) delay was $384 \pm 244$ days (Patient 1,4 months 10 days; Patient 2, 1 year 8 months 15 days; and Patient 3, 1 year 28 days). Two of the three patients had completed their antiplatelet protocol. One patient was taking only aspirin when the stroke occurred; the clopidogrel had been stopped as a result of delayed spontaneous hematoma on Day 14. All 3 patients were heavy smokers, and only 1 had stopped smoking after the procedure.

\section{Illustrative Cases}

\section{Patient 1}

\section{Presentation}

This 45-year-old man with a medical history of active smoking (60 pack-years), poorly controlled high blood pressure despite 4 antihypertensive treatments, and hypercholesterolemia presented with a subarachnoid hemorrhage in March 2007 (Glasgow Coma Scale Score 15). A ruptured saccular left superior cerebellar artery (SCA) aneurysm with a maximum diameter of $5.5 \mathrm{~mm}$ was diagnosed (Fig. 1).

\section{Treatment}

Initial endovascular treatment consisted of regular coiling (5 coils). The first recanalization was treated with balloon-assisted coiling (7 coils) in December 2012. A second recanalization was observed 6 months after the re-treatment. Finally, application of an FDS, the Pipeline embolization device (PED [Covidien]), was considered for treating this iterative recanalization, and the procedure was performed in February 2014. The patient began dual-antiplatelet therapy 5 days before stenting (clopidogrel and aspirin) and received full anticoagulation treatment (heparin) during the procedure. After the procedure, double antiaggregation was given, but on Day 14, the patient presented with a spontaneous left temporal hematoma and Wernicke aphasia, probably caused by a hypertensive peak; thus, clopidogrel therapy was interrupted. In June 2014, 3 episodes of transient diplopia caused by a probable aneurysmal sac thrombosis were reported.

\section{Very Late Stroke}

A delayed ischemic complication (perforator infarction type) occurred in July 2014 (136 days after stent placement), with a left paramedian pontine stroke found on DWI and suspected because of right cerebellar ataxia and moderate dysarthria (initial NIH Stroke Scale [NIHSS] Score 2). MRI also showed deep microbleeds, probably caused by hypertension (with no argument for cerebral amyloid angiopathy) and vascular leukopathy (Fazekas and Schmidt Grade 2 [data not shown]).

\section{Management}

Clopidogrel was reintroduced. In the acute phase of the stroke, no in-stent thrombosis was found on control DSA. Finally, the recovery of the patient was satisfactory with an NIHSS score of 1 on Day 7 and a modified Rankin Scale (mRS) score (at the time of this writing) of 2 (discrete dysarthria, seizure since temporal hematoma, and moderate cognitive impairment). A smoking-cessation program was initiated.

\section{Patient 2}

Presentation

This 53-year-old man with a medical history of smoking (2 packs/day, stopped in 2009), being overweight (body mass index $28.1 \mathrm{~kg} / \mathrm{m}^{2}$ ), and having hypercholesterolemia presented with a subarachnoid hemorrhage in January 2009 (Fisher Grade 4, World Federation of Neurosurgi- 

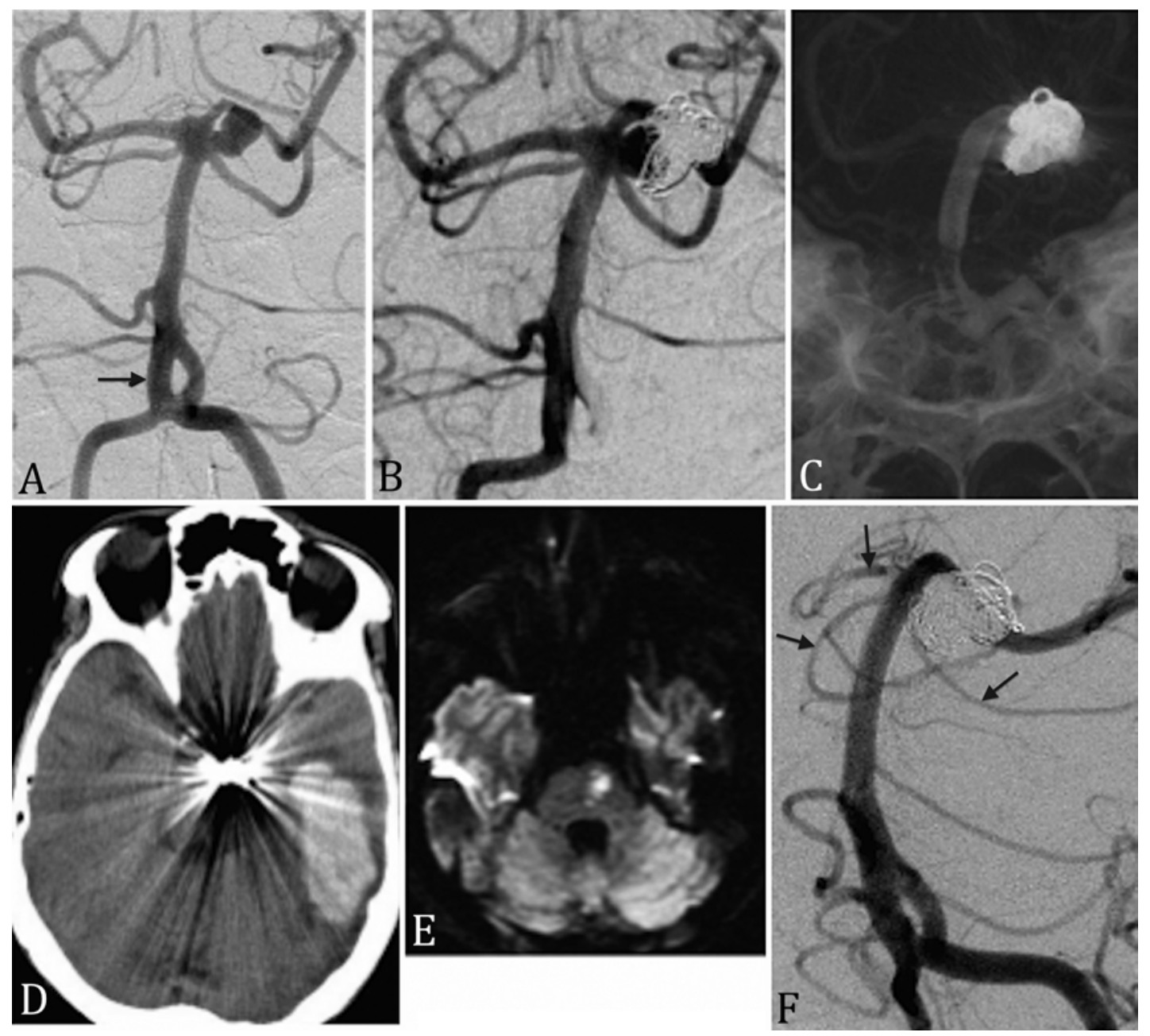

FIG. 1. Patient 1. Ruptured left SCA aneurysm in a 45-year-old man. A: Selective left vertebral artery DSA image, anteroposterior projection, showing a 5.5-mm saccular left SCA aneurysm. Note the proximal basilar artery fenestration (arrow). B: Control selective left vertebral artery DSA image, anteroposterior projection demonstrating second recanalization (Raymond-Roy Occlusion Class II) 6 years and 11 months after the first treatment. C: Contrast-enhanced flat-panel CT image obtained immediately after stent placement showing satisfactory stent deployment. D: Unenhanced axial CT image, acquired on Day 14, showing a left temporal hematoma. E: DWI image obtained 4 months after stent placement showing a left paramedian ischemic lesion of the pons caused by perforator infarction. F: Control DSA image of the vertebrobasilar system in the acute phase of the stroke; neither in-stent thrombosis nor misdeployment can be seen. Note the decreased flow inside the 2 SCAs and the right $P_{1}$ segment (arrows).

cal Societies Grade 5, Glasgow Coma Scale Score 5). A ruptured saccular right anterior choroidal artery (AChA) aneurysm with a maximum diameter of $15 \mathrm{~mm}$ was diagnosed (Fig. 2).

\section{Treatment}

Endovascular treatment was performed by regular coiling (23 coils). A recanalization occurred and was treated by an FDS (PED) in April 2010. The patient received dualantiplatelet therapy (clopidogrel and aspirin) 5 days before stent placement and full anticoagulation treatment (heparin) during the procedure. After the procedure, the double antiaggregation was continued for 3 months, and aspirin alone was used for 9 months. The protocol was followed to the end. Because of the initial subarachnoid hemorrhage, the patient presented related sequelae, such as cognitive impairment in executive functions.

\section{Very Late Stroke}

A delayed ischemic complication (side-wall covered branch infarction type) occurred in December 2011 (624 days after stent placement) that resulted in a right anterior choroidal stroke shown with DWI and suspected because of left hemiplegia, left hypoesthesia, left homonymous hemianopia, and oculomotor palsy (initial NIHSS Score $15)$.

\section{Management}

Intravenous thrombolysis (using recombinant tissue plasminogen activator [alteplase]) was performed on an emergency basis, and aspirin was introduced. The patient's postthrombolysis NIHSS score was 9. In the acute phase of the stroke, the right AChA was shown on DSA to be noncirculating. The patient progressively recovered after rehabilitation; at the time of this writing, his mRS score was 2 . 

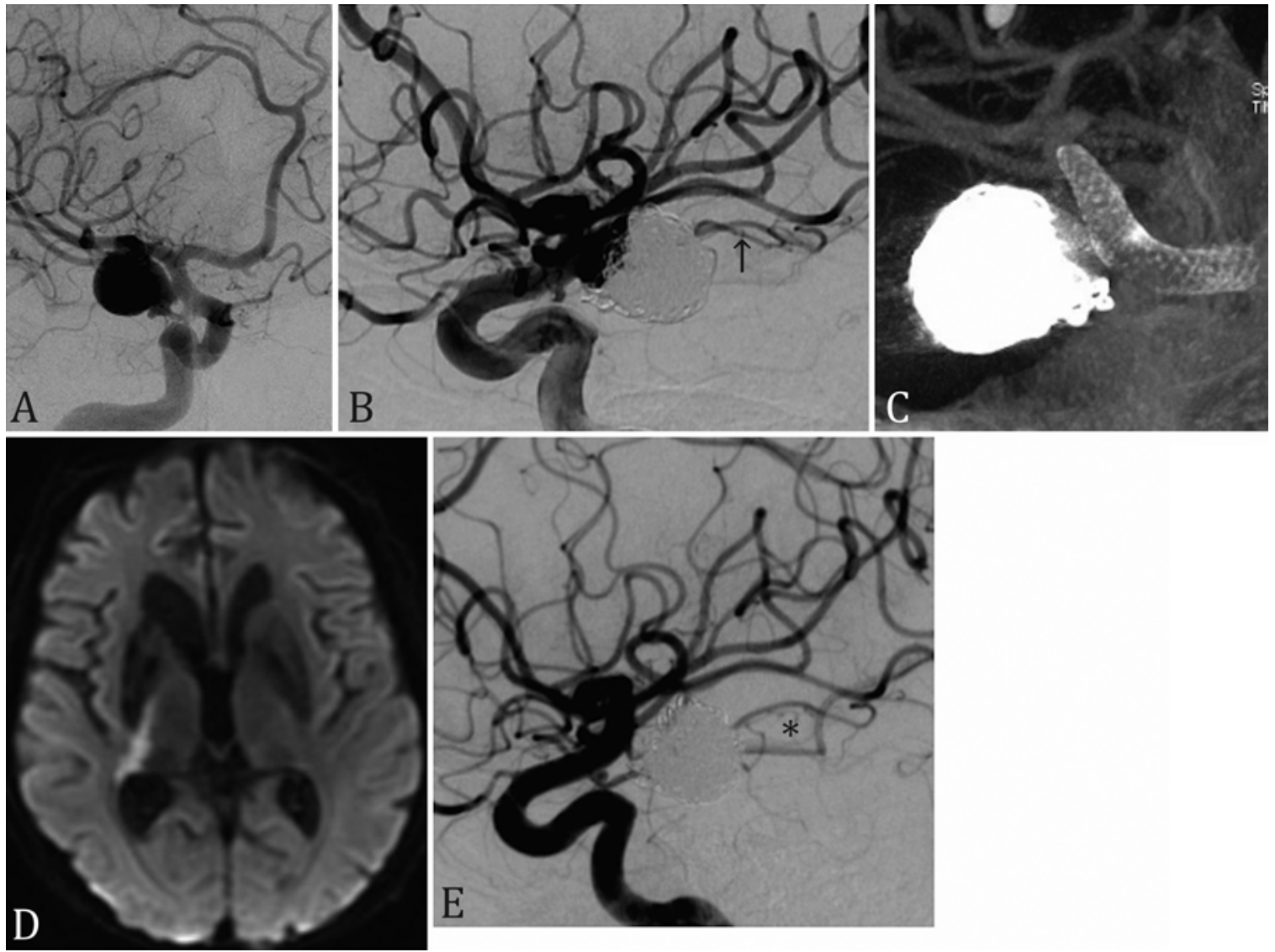

FIG. 2. Patient 2. Ruptured right $A C h A$ aneurysm in a 53-year-old man. A: Selective right internal carotid artery DSA image showing a saccular right AChA aneurysm (diameter $15 \mathrm{~mm}$ ). B: Control selective right internal carotid artery DSA image showing recanalization (Raymond-Roy Occlusion Class II) 15 months after the first treatment. Note that the right AChA was circulating (arrow). C: Contrast-enhanced flat-panel CT image obtained immediately after stent placement showing satisfactory stent deployment. D: DWI study acquired 20 months after stent placement showing a right AChA stroke (hyperintense signal of the posterior limb of the right internal capsule). E: Control right internal carotid artery DSA image in the acute phase of stroke showing a noncirculating right $\mathrm{AChA}$ (asterisk).

\section{Patient 3}

\section{Presentation}

This 37-year-old woman with a medical history of active smoking (9 pack-years) underwent an imaging workup for headaches in September 2013. An unruptured largenecked saccular left carotid-ophthalmic artery aneurysm with a maximum diameter of $9 \mathrm{~mm}$ was fortuitously diagnosed (Fig. 3).

\section{Treatment}

Initial endovascular treatment included placement of an FDS (PED) in October 2013. The patient received dualantiplatelet therapy (clopidogrel and aspirin) 5 days before stent placement and full anticoagulant treatment (heparin) during the procedure. After the procedure, double antiaggregation was given for 3 months, and aspirin alone was used for 9 months. The protocol was followed to the end.

\section{Very Late Stroke}

A delayed ischemic complication (thromboembolic type) occurred in November 2014 (393 days after stent placement) and a superficial and deep left middle artery territory stroke was seen on DWI, which had been suspected because of right hemiapraxia and slight word missing (initial NIHSS Score 2). The symptoms gradually appeared over 2 days.

\section{Management}

Aspirin therapy was resumed when the diagnosis of acute ischemic stroke was confirmed with DWI. The NIHSS score at 24 hours was 0 . In the acute phase of the stroke, DSA revealed a distal focal stenosis (approximately $40 \%$ ) at the distal aspect of the stent, consistent with a probable intimal hyperplasia. It is noteworthy that the patient was taking an oral hormonal contraceptive (secondgeneration pills with $100 \mu \mathrm{g}$ of levonorgestrel and $20 \mu \mathrm{g}$ of ethinyl estradiol) and continued smoking at the time of the stroke. She was given double antiaggregation (aspirin and clopidogrel) and nicotine substitutes; oral contraception was stopped, and an intrauterine device was considered. At the time of this writing, the patient was asymptomatic (mRS Score 0). Control DSA at the 6-month followup revealed improvement of the stenosis. 


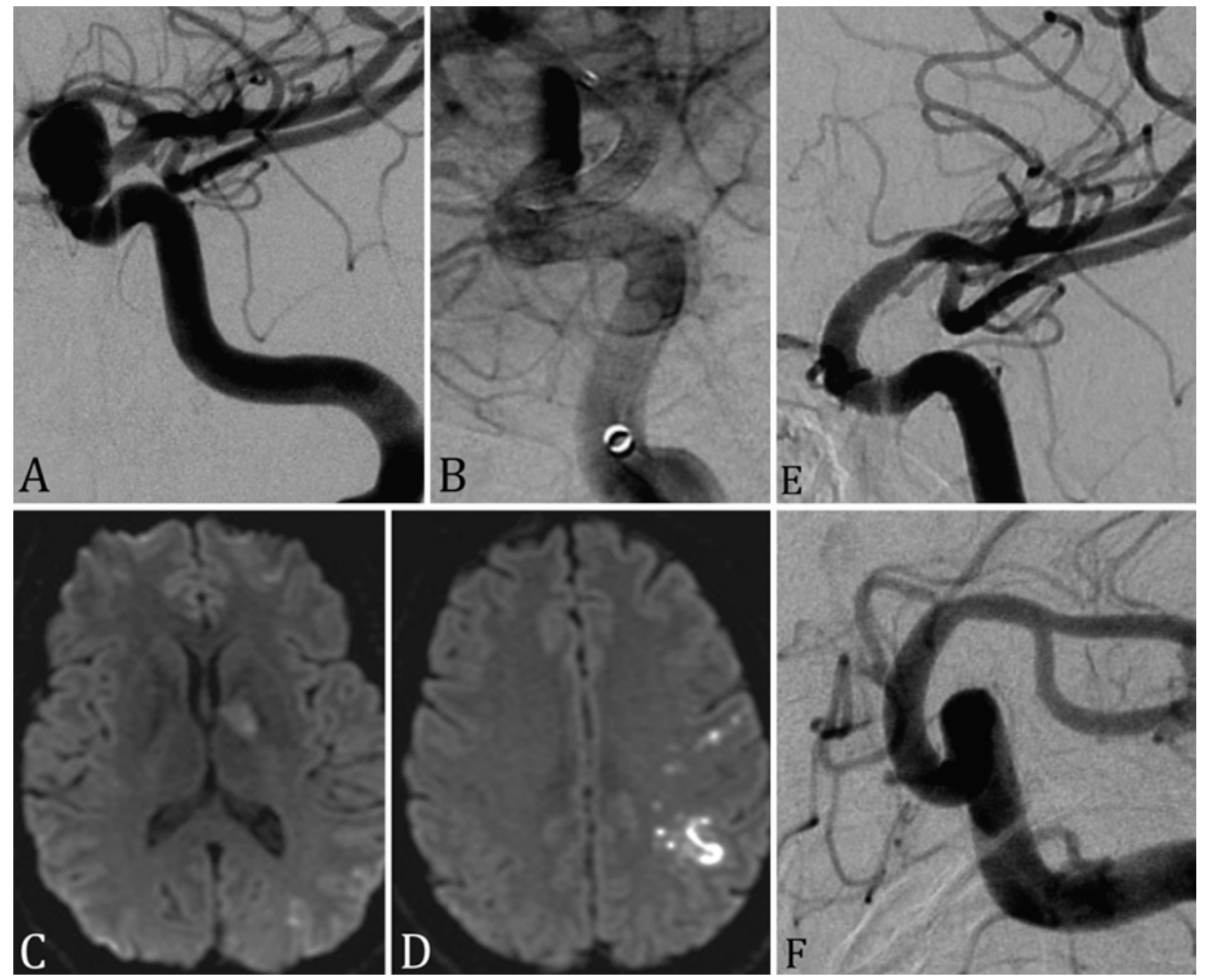

FIG. 3. Patient 3. Unruptured left carotid-ophthalmic artery aneurysm in a 37-year-old woman. A: Selective left internal carotid artery DSA image demonstrating a saccular left carotid-ophthalmic artery aneurysm (diameter $9 \mathrm{~mm}$ ). B: Unsubtracted control DSA image obtained immediately after FDS placement showing satisfactory stent deployment. Note the stagnation of the contrast medium inside the aneurysm's sac. C and D: DWI studies acquired 13 months after stent placement showing a thromboembolic stroke in the superficial and deep left middle artery territories. E: Left internal carotid artery DSA control image in the acute phase of stroke showing a focal stenosis at the distal aspect of the FDS ( $40 \%)$. F: Control left internal carotid artery DSA image obtained 6 months after the ischemic stroke showing significant regression of the stenosis.

\section{Discussion}

The rate of very late ( $\geq 4$-month) ischemic complications in our institution during the study period was $3.5 \%$ (3 of 86) with an average delay of 384 days (range 136624 days) after the procedure. Note that the overall rate of ischemic complications was 5.8\% (5 of 86), similar to that found in a recent meta-analysis ${ }^{5}$ (total ischemic stroke rate $6 \%$ ).

The patency of the perforating arteries and side-wall branches covered by the device is an important issue in the treatment of intracranial aneurysm using an FDS. Indeed, the sudden flow decrease may be responsible for stroke, as in Patient 1 (perforator infarction type at 4 months) and Patient 2 (side-wall covered branch infarction type at 1 year 8 months). The use of such high-structural-profile implants raises the question of safety, because the mesh is much denser than that of conventional stents (e.g., 30\%$35 \%$ of metal surface coverage for the PED ${ }^{12}$ ). Thus, FDSs reduce blood flow in the aneurysm sac to the point of stagnation and thrombosis, as demonstrated in experimental ${ }^{21}$ and animal ${ }^{24}$ studies. Furthermore, according to the literature, FDSs induce the development of the neointima in the inner surface of the vessel with a complete wall recon- struction while preserving patency of the adjacent small vessels. ${ }^{16}$ The existing data (derived from the cardiology literature on coronary arteries) suggest that intimal hyperplasia peaks at 3-4 months ${ }^{14}$ and very rarely progresses beyond 6 months after bare-metal-stent implantation. ${ }^{17}$

On Day 14, Patient 1 experienced a spontaneous left temporal hematoma. Systemic hypertension is not the underlying cause in most nontraumatic lobar hematomas (18\% according to Loes et al. ${ }^{22}$ ). However, in the acute phase of the hematoma, Patient 1 was still under medical supervision for poorly controlled hypertension. If a patient is receiving dual-antiplatelet therapy, a systolic blood pressure peak beyond $200 \mathrm{~mm} \mathrm{Hg}$ may precipitate spontaneous bleeding. The initial clinical symptoms and delayed MRI were not suggestive of ischemic stroke with hemorrhagic transformation. Note that previous DSA had not revealed vascular malformation on the left temporal lobe. Delayed ipsilateral parenchymal hemorrhage (DIPH) cannot be excluded in this case. Indeed, DIPHs have been reported as spontaneous hematomas from unclear origin distant from the aneurysm treated with an FDS and, most of the time, on the ipsilateral side. ${ }^{27}$ These DIPHs can occur up to 1 week $^{7}$ or even 2 weeks ${ }^{2}$ after treatment. 
Because of the hematoma, Patient 1 was taking only aspirin when the stroke occurred, and tiny thrombi could have formed and covered the orifice of the perforating branches of the basilar artery. However, 4 months after its placement, the device has usually been incorporated into the vessel wall, which reduces its thrombogenicity. Therefore, neointimal overgrowth and progressive narrowing of the perforator's orifice should also be considered as potential causal factors. In a recent meta-analysis, ${ }^{5}$ ischemic stroke and perforator infarction were reported at the significantly highest rate among patients with posterior circulation aneurysm because of delicate perfusion and lack of collaterals to brainstem structures. Kulcsár et al. ${ }^{19}$ reported a case of very late thalamic infarct developed 7 months after FDS placement for a basilar artery aneurysm. In this case, the patient was taking only aspirin at the time of the stroke. The mRS score at latest follow-up was 4. The authors questioned the neointimal overgrowth and progressive narrowing of the perforator's orifice, especially because 2 Neuroform stents (Stryker) had been implanted earlier. The safety and indication of FDSs in the posterior system are still controversial. ${ }^{28}$

In Patient 2, the right AChA covered by the stent was noncirculating during the acute ischemic complication despite 1 year of antiplatelet therapy. In a recent series of 15 aneurysms in which a PED was placed across the ostium of the AChA, Brinjikji et al. ${ }^{4}$ reported an AChA occlusion rate of 7\% (1 of 14) without any acute ischemic stroke (with follow-up of at least 6 months). Another recent retrospective study ${ }^{13}$ of 59 patients reported 2 cases of asymptomatic delayed occlusion of the ophthalmic artery covered by a PED without other-side branch occlusion and a progressive arterial narrowing rate of $16.2 \%$ according to 12-month follow-up DSA. Moreover, in Patient 2, partial efficacy of thrombolysis with a reduction in the NIHSS score suggests a thromboembolic factor.

It is also interesting to note that for Patient 1, FDS placement was the third treatment (second recanalization after 2 aneurysm sac exclusions with Raymond-Roy Occlusion Classification [RROC] I) for the aneurysm (with previous balloon-assisted coiling re-treatment), and it was the second treatment for Patient 2; thus, a part of vasculopathy may play a role in very late ischemic complications.

In Patient 3, control DSA of the carotid artery in the acute phase of stroke revealed a distal focal stenosis estimated at $40 \%$. There was satisfactory stent deployment during the initial treatment, and the stent was in place during late DSA. Indeed, cases of very delayed stroke have been reported secondary to mechanical causes (e.g., distal migration of a PED at 5 months, ${ }^{6}$ incomplete wall apposition of the stent with clot formation at 4 months caused by slow blood flow at the vessel wall and implant interface ${ }^{10}$ ). Reactive in-stent stenosis seems to be a frequent (38\% at 2 months' follow-up ${ }^{8}$ ) asymptomatic and early angiographic finding. It may play a fundamental role in the process of stent-inducing aneurysm occlusion. Lubicz et al..$^{23}$ reported a case of very delayed infarct at 4 months with intrastent stenosis at the time of the intimal hyperplasia peak. However, a late occurrence of stenosis, as seen in Patient 3 ( $>1$ year later), is not usual.

In our study, 2 patients were active smokers at the time of their very late ischemic complication after FDS placement. There is considerable scientific evidence that proves a strong dose-response relationship between smoking and stroke risk. Indeed, smoking increases the risk of stroke by three- to fourfold. ${ }^{25}$ It is important to emphasize to the patient the need to stop or reduce smoking and to help all patients quit smoking with a specific cessation program. Likewise, in 37-year-old Patient 3, oral hormonal contraception associated with smoking was a risk factor for stroke. Indeed, the risk of thrombotic stroke is increased by a factor of $0.9-1.7$ with oral contraceptives, including ethinyl estradiol at a dose of $20 \mu \mathrm{g} .{ }^{20}$ It is likely that taking care and following-up on all thrombotic risk factors for patients with an FDS could prevent very delayed ischemic complications.

No very late ischemic complications were seen among patients who were receiving dual-antiplatelet therapy (aspirin and clopidogrel). Medical management is essential after FDS deployment to avoid possible platelet aggregation on the stent walls or occlusion of the smallest collateral vessels covered by the stent. Delayed stroke resulting from in-stent thrombosis has been reported (e.g., at 23 months ${ }^{11}$ and at 12 months $^{18}$ ) with the patient on aspirin only. The need for antiplatelet therapy before, during, and after the endovascular procedure should be clearly explained to patients along with the risk of serious hemorrhagic or thromboembolic events. There is a lack of evidence regarding the idea duration of dual-antiplatelet therapy. A recent meta-analysis ${ }^{5}$ failed to analyze the influence of the antiplatelet therapy regimen on clinical complications, because a majority of the studies pooled did not stratify clinical outcomes on this variable.

However, according to Alderazi et al., dual-antiplatelet therapy has been continued until 6 months after the procedure in most studies. Aspirin is typically continued for at least 12 months, whereas clopidogrel may be stopped depending on angiographic and clinical results. In published series, thromboembolic complications, including in-stent thrombosis, have often occurred after stopping clopidogrel (e.g., in a De Vries et al. study ${ }^{10}$ after discontinuation of clopidogrel at 3 months). Recently, Skukalek et al. ${ }^{26}$ showed that, after PED placement, high-dose aspirin ( $\geq$ $300 \mathrm{mg}$ ) for $>6$ months is associated with fewer permanent thrombotic and hemorrhagic events, and clopidogrel therapy for $\leq 6$ months is associated with higher rates of thrombotic events. In contrast, aspirin treatment has been reported to be associated with an absolute risk increase in hemorrhagic stroke of 1.2 events per 1000 persons..$^{15} \mathrm{In}$ a stroke trial that involved 3020 patients, the risk of major hemorrhage was almost doubled with dual-antiplatelet therapy ( $2.1 \%$ per year) over that with aspirin alone $(1.1 \%$ per year). ${ }^{3}$ Moreover, the use of routine platelet-function testing is still not consensual. ${ }^{9}$ The durations of treatment have not been homogeneous in the literature, and studies to clarify this issue are needed, especially to determine the right moment at which to stop clopidogrel (based on the characteristics of the aneurysm and treatment) and if the overall benefit of long-term aspirin use may outweigh its adverse effects. However, the results of our study suggest pursuing longer antiplatelet therapy, at least in selected patients with vascular risk factors. 


\section{Conclusions}

Interventional neuroradiologists should be aware of very late ischemic complications after FDS treatment. They were observed in $3.5 \%$ of the cases in our series and may occur as late as more than 1 year after treatment. Our 3 cases underscore the need for continued and careful evaluation and close long-term follow-up of patients treated with this new device.

\section{References}

1. Alderazi YJ, Shastri D, Kass-Hout T, Prestigiacomo CJ, Gandhi CD: Flow diverters for intracranial aneurysms. Stroke Res Treat 2014:415653, 2014

2. Becske T, Kallmes DF, Saatci I, McDougall CG, Szikora I, Lanzino G, et al: Pipeline for uncoilable or failed aneurysms: results from a multicenter clinical trial. Radiology 267:858868,2013

3. Benavente OR, Hart RG, McClure LA, Szychowski JM, Coffey CS, Pearce LA: Effects of clopidogrel added to aspirin in patients with recent lacunar stroke. N Engl J Med 367:817825,2012

4. Brinjikji W, Kallmes DF, Cloft HJ, Lanzino G: Patency of the anterior choroidal artery after flow-diversion treatment of internal carotid artery aneurysms. AJNR Am J Neuroradiol 36:537-541, 2015

5. Brinjikji W, Murad MH, Lanzino G, Cloft HJ, Kallmes DF: Endovascular treatment of intracranial aneurysms with flow diverters: a meta-analysis. Stroke 44:442-447, 2013

6. Chalouhi N, Tjoumakaris SI, Gonzalez LF, Hasan D, Pema PJ, Gould G, et al: Spontaneous delayed migration/shortening of the Pipeline embolization device: report of 5 cases. AJNR Am J Neuroradiol 34:2326-2330, 2013

7. Clarençon F, Di Maria F, Biondi A, Chiras J, Sourour NA: Distant and delayed ( $>7$ days) hemorrhage after treatment by flow-diverter stents in intracranial aneurysms: a rare but potentially serious complication. AJNR Am J Neuroradiol 34:E81-E82, 2013

8. Cohen JE, Gomori JM, Moscovici S, Leker RR, Itshayek E: Delayed complications after flow-diverter stenting: reactive in-stent stenosis and creeping stents. J Clin Neurosci 21:1116-1122, 2014

9. Comin J, Kallmes DF: Platelet-function testing in patients undergoing neurovascular procedures: caught between a rock and a hard place. AJNR Am J Neuroradiol 34:730-734, 2013

10. De Vries J, Boogaarts J, Van Norden A, Wakhloo AK: New generation of Flow Diverter (surpass) for unruptured intracranial aneurysms: a prospective single-center study in 37 patients. Stroke 44:1567-1577, 2013

11. Fiorella D, Hsu D, Woo HH, Tarr RW, Nelson PK: Very late thrombosis of a Pipeline embolization device construct: case report. Neurosurgery 67 (3 Suppl Operative):onsE313onsE314, 2010

12. Fiorella D, Woo HH, Albuquerque FC, Nelson PK: Definitive reconstruction of circumferential, fusiform intracranial aneurysms with the Pipeline embolization device. Neurosurgery 62:1115-1121, 2008

13. Gascou G, Lobotesis K, Brunel H, Machi P, Riquelme C, Eker O, et al: Extra-aneurysmal flow modification following Pipeline embolization device implantation: focus on regional branches, perforators, and the parent vessel. AJNR Am J Neuroradiol 36:725-731, 2015

14. Grewe PH, Deneke T, Machraoui A, Barmeyer J, Müller K-M: Acute and chronic tissue response to coronary stent implantation: pathologic findings in human specimen. J Am Coll Cardiol 35:157-163, 2000

15. He J, Whelton PK, Vu B, Klag MJ: Aspirin and risk of hem- orrhagic stroke: a meta-analysis of randomized controlled trials. JAMA 280:1930-1935, 1998

16. Kallmes DF, Ding YH, Dai D, Kadirvel R, Lewis DA, Cloft HJ: A new endoluminal, flow-disrupting device for treatment of saccular aneurysms. Stroke 38:2346-2352, 2007

17. Kimura T, Yokoi H, Nakagawa Y, Tamura T, Kaburagi S, Sawada Y, et al: Three-year follow-up after implantation of metallic coronary-artery stents. N Engl J Med 334:561-566, 1996

18. Klisch J, Turk A, Turner R, Woo HH, Fiorella D: Very late thrombosis of flow-diverting constructs after the treatment of large fusiform posterior circulation aneurysms. AJNR Am J Neuroradiol 32:627-632, 2011

19. Kulcsár Z, Ernemann U, Wetzel SG, Bock A, Goericke S, Panagiotopoulos V, et al: High-profile flow diverter (silk) implantation in the basilar artery: efficacy in the treatment of aneurysms and the role of the perforators. Stroke 41:16901696, 2010

20. Lidegaard Ø, Løkkegaard E, Jensen A, Skovlund CW, Keiding $\mathrm{N}$ : Thrombotic stroke and myocardial infarction with hormonal contraception. N Engl J Med 366:2257-2266, 2012

21. Lieber BB, Stancampiano AP, Wakhloo AK: Alteration of hemodynamics in aneurysm models by stenting: influence of stent porosity. Ann Biomed Eng 25:460-469, 1997

22. Loes DJ, Smoker WRK, Biller J, Cornell SH: Nontraumatic lobar intracerebral hemorrhage: CT/angiographic correlation. AJNR Am J Neuroradiol 8:1027-1030, 1987

23. Lubicz B, Collignon L, Raphaeli G, Pruvo JP, Bruneau M, De Witte O, et al: Flow-diverter stent for the endovascular treatment of intracranial aneurysms: a prospective study in 29 patients with 34 aneurysms. Stroke 41:2247-2253, 2010

24. Sadasivan C, Cesar L, Seong J, Rakian A, Hao Q, Tio FO, et al: An original flow diversion device for the treatment of intracranial aneurysms: evaluation in the rabbit elastaseinduced model. Stroke 40:952-958, 2009

25. Shah RS, Cole JW: Smoking and stroke: the more you smoke the more you stroke. Expert Rev Cardiovasc Ther 8:917932, 2010

26. Skukalek SL, Winkler AM, Kang J, Dion JE, Cawley CM, Webb A, et al: Effect of antiplatelet therapy and platelet function testing on hemorrhagic and thrombotic complications in patients with cerebral aneurysms treated with the Pipeline embolization device: a review and meta-analysis. J Neurointerv Surg [epub ahead of print], 2014

27. Tomas C, Benaissa A, Herbreteau D, Kadziolka K, Pierot L: Delayed ipsilateral parenchymal hemorrhage following treatment of intracranial aneurysms with flow diverter. Neuroradiology 56:155-161, 2014

28. van Rooij WJ: Flow diverters for unruptured aneurysms: are they safe enough? Neuroradiology 54:1179-1182, 2012 (Letter)

\section{Disclosures}

Dr. Sourour is proctor for the PED (ev3/Covidien) and Dr. Biondi is a consultant for ev3/Covidien.

\section{Author Contributions}

Conception and design: Sourour, Guédon, Clarençon. Acquisition of data: Guédon, Gabrieli. Analysis and interpretation of data: Guédon, Di Maria, Rosso, Biondi, Rojas. Drafting the article: Guédon. Critically revising the article: Sourour, Clarençon, Chiras. Reviewed submitted version of manuscript: Sourour, Clarençon, Chiras. Approved the final version of the manuscript on behalf of all authors: Sourour.

\section{Correspondence}

Nader Sourour, Department of Interventional Neuroradiology, Pitié-Salpêtrière Hospital, 47 Blvd. de l'Hôpital, Paris 75013, France.email: nsourour@gmail.com. 